\title{
Nocardioides insulae sp. nov., isolated from soil
}

Correspondence

Jung-Hoon Yoon

jhyoon@kribb.re.kr

\section{Jung-Hoon Yoon, So-Jung Kang, Choong-Hwan Lee and Tae-Kwang Oh}

Korea Research Institute of Bioscience and Biotechnology (KRIBB), PO Box 115, Yusong,

Taejon, Korea
The genus Nocardioides was proposed by Prauser (1976) and, at the time of writing, comprises 18 recognized species, including the recently described species Nocardioides lentus (Yoon et al., 2006a) and Nocardioides kongjuensis (Yoon et al., 2006b). Here, we report on the taxonomic characterization of a Nocardioides-like strain, DS- $51^{\mathrm{T}}$, which was isolated from a soil collected in Dokdo, an island of Korea.

Strain DS $-51^{\mathrm{T}}$ was isolated by using the standard dilution plating technique at $30^{\circ} \mathrm{C}$ on $10 \times$ diluted nutrient agar (NA; Difco). To investigate its morphological, physiological and biochemical characteristics, strain DS- $51^{\mathrm{T}}$ was routinely cultivated at $30^{\circ} \mathrm{C}$ on NA and in nutrient broth (NB; Difco). The morphological, physiological, cultural and biochemical properties were examined as described by Yoon et al. (2005a). Growth at various $\mathrm{NaCl}$ concentrations $(0-8 \%$, $\mathrm{w} / \mathrm{v}$, using increments of $1.0 \%$ ) was investigated in trypticase soy broth prepared according to the formula of Difco medium except that no $\mathrm{NaCl}$ was used. The $\mathrm{pH}$ range for growth was determined in NB that was adjusted to various $\mathrm{pH}$ values ( $\mathrm{pH} 4.0-10.5$, using increments of $0.5 \mathrm{pH}$ units) by the addition of $\mathrm{HCl}$ and $\mathrm{Na}_{2} \mathrm{CO}_{3}$. The susceptibility of strain DS- $51^{\mathrm{T}}$ to various antibiotics was tested on NA plates by using antibiotic discs, as follows: $100 \mathrm{U}$ polymyxin $\mathrm{B}$, $50 \mu \mathrm{g}$ streptomycin, $20 \mathrm{U}$ penicillin $\mathrm{G}, 100 \mu \mathrm{g}$ chloramphenicol, $10 \mu \mathrm{g}$ ampicillin, $30 \mu \mathrm{g}$ cephalothin, $30 \mu \mathrm{g}$ gentamicin, $5 \mu \mathrm{g}$ novobiocin, $30 \mu \mathrm{g}$ tetracycline, $30 \mu \mathrm{g}$

The GenBank/EMBL/DDBJ accession number for the 16S rRNA gene sequence of strain DS- $51^{\top}$ is DQ786794. kanamycin, $15 \mu \mathrm{g}$ lincomycin, $15 \mu \mathrm{g}$ oleandomycin, $30 \mu \mathrm{g}$ neomycin and $100 \mu \mathrm{g}$ carbenicillin. Other physiological and biochemical properties were tested by using the API 20E and API ZYM systems (bioMérieux). Cell biomass for DNA extraction and for analyses of cell walls and isoprenoid quinones was obtained by cultivation in $\mathrm{NB}$ at $30^{\circ} \mathrm{C}$ for 3 days. For fatty acid methyl ester analysis, cell mass of strain DS- $51^{\mathrm{T}}$ was harvested from NA plates after incubation at $30^{\circ} \mathrm{C}$ for 7 days. Chemotaxonomic and molecular systematic studies were performed as described by Yoon et al. (2005a). The isomer type of the diamino acid in the cell-wall peptidoglycan was analysed using TLC according to the method described by Komagata \& Suzuki (1987).

Morphological, cultural, physiological and biochemical characteristics of strain DS $-51^{\mathrm{T}}$ are given in the species description (see below) or are shown in Table 1. Strain DS$51^{\mathrm{T}}$ was found to be susceptible to polymyxin $\mathrm{B}$, streptomycin, chloramphenicol, cephalothin, gentamicin, tetracycline, carbenicillin, kanamycin, lincomycin, neomycin and oleandomycin, but not to ampicillin, novobiocin or penicillin G. The almost-complete $16 \mathrm{~S}$ rRNA gene sequence of strain DS-51 ${ }^{\mathrm{T}}$, comprising $1484 \mathrm{nt}$ (approx. $96 \%$ of the Escherichia coli $16 \mathrm{~S}$ rRNA gene sequence), was determined in this study. Comparative 16S rRNA gene sequence analyses showed that strain DS- $51^{\mathrm{T}}$ is most closely affiliated to the genus Nocardioides (Fig. 1). In the neighbour-joining phylogenetic tree based on 16S rRNA gene sequences, strain DS- $51^{\mathrm{T}}$ formed a distinct phylogenetic lineage with the radiation of the cluster comprising Nocardioides species (Fig. 1). Strain DS $-51^{\mathrm{T}}$ exhibited $16 \mathrm{~S}$ rRNA gene sequence 
Table 1. Differential phenotypic characteristics of strain DS- $51^{\top}$ and other Nocardioides species

Taxa: 1, strain DS-51 ${ }^{\text {T }} ; 2$, N. albus; 3, N. luteus; 4, N. simplex; 5, N. plantarum; 6, N. pyridinolyticus; 7, N. nitrophenolicus; 8, N. aquaticus; 9, N. aquiterrae; 10, N. ganghwensis; 11, N. aestuarii; 12, N. alkalitolerans; 13, N. kribbensis; 14, N. oleivorans; 15, N. aromaticivorans; 16, N. lentus; 17, N. kongjuensis. Data are from Collins et al. (1989, 1994), Lawson et al. (2000), Prauser (1976, 1984, 1989), Suzuki \& Komagata (1983), Yoon et al. (1997, 1999, 2004, 2005a, b, 2006a, b), Yi \& Chun, (2004a, b), Schippers et al. (2005) and Kubota et al. (2005). +, Positive reaction; -, negative reaction; ND, not determined; w, weakly positive reaction; $\mathrm{v}$, variable reaction. Data in parentheses are for the type strain. All species are positive for the Gram stain, for catalase and for esterase lipase (C8) (not determined for $N$. oleivorans). All species are negative for $\beta$-glucuronidase, $N$-acetyl- $\beta$-glucosaminidase and $\alpha$-fucosidase activities (not determined for N. oleivorans).

\begin{tabular}{|c|c|c|c|c|c|c|c|c|c|c|c|c|c|c|c|c|c|}
\hline Characteristic & 1 & 2 & 3 & 4 & 5 & 6 & 7 & 8 & 9 & 10 & 11 & 12 & 13 & 14 & 15 & 16 & 17 \\
\hline Cell morphology & $\begin{array}{l}\text { Rods, } \\
\text { cocci }\end{array}$ & Hyphae & Hyphae & Rods, cocci & $\begin{array}{c}\text { Short } \\
\text { rods, cocci }\end{array}$ & $\begin{array}{l}\text { Rods, } \\
\text { cocci }\end{array}$ & $\begin{array}{l}\text { Rods, } \\
\text { cocci }\end{array}$ & $\begin{array}{c}\text { Cocci, } \\
\text { short rods }\end{array}$ & $\begin{array}{l}\text { Rods, } \\
\text { cocci }\end{array}$ & Rods & Rods & $\begin{array}{l}\text { Rods, } \\
\text { cocci }\end{array}$ & $\begin{array}{c}\text { Short } \\
\text { rods, cocci }\end{array}$ & $\begin{array}{l}\text { Irregular } \\
\text { rods }\end{array}$ & Rods & Rods, cocci & Rods, cocci \\
\hline Cell size $(\mu \mathrm{m})$ & $\begin{array}{r}0.6-1.0 \times \\
1.3-6.0\end{array}$ & $0.5-1.0^{\star}$ & $0.5-1.0^{\star}$ & $\begin{array}{c}1.0-1.2 \times \\
1.5-6.0\end{array}$ & ND & $\begin{array}{r}0.5-0.6 \times \\
1.2-1.6\end{array}$ & $\begin{array}{r}0.5-0.8 \times \\
1.0-3.0\end{array}$ & $\begin{array}{c}0.9-1.0 \times \\
0.9-1.4\end{array}$ & $\begin{array}{r}0.8-1.0 \times \\
1.7-2.0\end{array}$ & $\begin{array}{c}0.4-0.5 \times \\
0.9-4.5\end{array}$ & $\begin{array}{c}0.3-0.4 \times \\
0.9-2.1\end{array}$ & $\begin{array}{r}0.8-1.0 \times \\
1.5-2.0\end{array}$ & $\begin{array}{c}0.8-1.0 \times \\
1.5-2.0\end{array}$ & $0.3 \times \sim 1.1$ & $\begin{array}{r}0.5-0.7 \times \\
1.0-2.0\end{array}$ & $\begin{array}{c}0.4-0.7 \times \\
1.0-4.5\end{array}$ & $\begin{array}{c}0.4-0.7 \times \\
0.8-3.0\end{array}$ \\
\hline Motility & - & - & - & + & - & + & + & - & + & - & - & - & - & - & - & - & - \\
\hline Colony colour $\dagger$ & Ivory & $\begin{array}{l}\text { Whitish to } \\
\text { faintly } \\
\text { brownish }\end{array}$ & $\begin{array}{l}\text { Yellow to } \\
\text { orange-yellow } \\
\text { or cream }\end{array}$ & $\begin{array}{l}\text { Yellowish } \\
\text { white }\end{array}$ & ND & Cream & $\begin{array}{l}\text { Yellowish } \\
\text { white }\end{array}$ & Dull orange & Cream & Ivory & Ivory & $\begin{array}{l}\text { Milky } \\
\text { white }\end{array}$ & Cream & Orange & $\begin{array}{l}\text { Milky } \\
\text { white }\end{array}$ & Yellow & $\begin{array}{l}\text { Yellowish } \\
\text { white }\end{array}$ \\
\hline $\begin{array}{l}\text { Optimum } \\
\text { temperature }\left({ }^{\circ} \mathrm{C}\right)\end{array}$ & 30 & 28 & 28 & $26-37$ & 25 & 35 & 30 & $16-26$ & 30 & 30 & 30 & $25-30$ & 30 & 30 & 30 & 28 & $30-37$ \\
\hline Nitrate reduction & + & $(-)$ & - & $(-)$ & - & + & - & + & + & + & - & + & + & $\mathrm{ND}$ & - & + & - \\
\hline \multicolumn{18}{|l|}{ Hydrolysis of: } \\
\hline Aesculin & - & (w) & + & $(+)$ & $\mathrm{w}$ & + & + & - & + & w & w & - & + & $\mathrm{ND}$ & + & - & - \\
\hline Casein & + & $(+)$ & + & + & $+\ddagger$ & + & + & + & + & + & + & + & + & $\mathrm{ND}$ & + & + & + \\
\hline Gelatin & + & $(+)$ & + & + & + & + & + & + & + & + & + & $\mathrm{v}(+)$ & + & $\mathrm{ND}$ & $\mathrm{ND}$ & + & + \\
\hline Hypoxanthine & - & $(+)$ & + & $(-)$ & - & - & - & - & - & - & - & - & - & $\mathrm{ND}$ & + & - & - \\
\hline Starch & - & $(+)$ & + & (w) & - & + & + & $\mathrm{w} \ddagger$ & - & + & - & - & - & $\mathrm{ND}$ & - & - & - \\
\hline Tween 80 & + & $(+)$ & + & $(+)$ & + & - & + & + & + & + & + & + & + & $\mathrm{ND}$ & - & + & + \\
\hline Tyrosine & - & $(+)$ & + & $(+)$ & - & + & + & + & - & + & - & + & + & ND & - & $\mathrm{v}(+)$ & - \\
\hline Urea & - & $(-)$ & - & - & - & - & + & - & - & - & - & - & - & $\mathrm{ND}$ & + & - & - \\
\hline Xanthine & - & $(+)$ & $-\ddagger$ & $(-)$ & - & + & - & - & - & w & - & - & - & $\mathrm{ND}$ & - & - & - \\
\hline \multicolumn{18}{|c|}{ Utilization as sole carbon and energy sources: } \\
\hline L-Arabinose & - & + & + & - & - & - & - & - & - & + & - & + & - & - & + or $\mathrm{W}$ & $\mathrm{w}$ & - \\
\hline D-Cellobiose & + & $(+)$ & + & $(-)$ & + & + & - & - & + & + & + & + & + & + & + & + & - \\
\hline D-Fructose & - & + & $+\neq$ & $(-)$ & + & + & + & + & + & + & + & - & - & + & + & - & - \\
\hline D-Galactose & + & $(+)$ & - & $(-)$ & - & + & - & $-\ddagger$ & + & + & + & - & + & + & - & + & - \\
\hline D-Glucose & + & + & + & + & $+\ddagger$ & + & + & + & + & + & + & - & + & + & + & + & + \\
\hline Maltose & $\mathrm{w}$ & $\mathrm{ND}$ & $\mathrm{ND}$ & ND & + & + & - & $\mathrm{w}$ & + & ND & $\mathrm{ND}$ & $\mathrm{v}(-)$ & + & + & + or $\mathrm{w}$ & + & - \\
\hline D-Mannose & $\mathrm{w}$ & $(+)$ & + & $(-)$ & - & $+\ddagger$ & $\mathrm{w} \neq$ & - & + & + & - & - & - & + & $\mathrm{w}$ & - & $\mathrm{w}$ \\
\hline Sucrose & - & $\mathrm{v}(+\ddagger)$ & $+末$ & + & + & + & + & + & + & + & + & - & + & + & + & + & + \\
\hline D-Trehalose & + & ND & $\mathrm{ND}$ & ND & + & + & + & w & + & ND & ND & + & + & + & ND & + & + \\
\hline D-Xylose & $\mathrm{w}$ & + & + & - & + & + & + & $\mathrm{w} \ddagger$ & + & + & + & - & - & ND & + & $\mathrm{v}(+)$ & - \\
\hline \multicolumn{18}{|l|}{ API ZYM tests: } \\
\hline Acid phosphatase & + & $(-)$ & - & (w) & $\mathrm{w}$ & + & + & + & + & w & w & + & + & $\mathrm{ND}$ & - & + & + \\
\hline Alkaline phosphatase & + & $(+)$ & $\mathrm{v}$ & $(+)$ & - & + & + & $\mathrm{v}$ & - & + & $\mathrm{w}$ & + & + & $\mathrm{ND}$ & + & + & + \\
\hline$\alpha$-Chymotrypsin & - & $(-)$ & - & $(-)$ & - & - & - & - & - & - & + & - & + & $\mathrm{ND}$ & - & - & - \\
\hline Cystine arylamidase & - & $(-)$ & - & (w) & + & - & w & $\mathrm{v}$ & $\mathrm{w}$ & w & - & - & - & $\mathrm{ND}$ & - & - & - \\
\hline Esterase (C4) & + & $(+)$ & + & $(-)$ & + & - & - & w & - & $\mathrm{v}$ & + & + & + & $\mathrm{ND}$ & - & + & - \\
\hline$\alpha$-Galactosidase & - & $(-)$ & - & $(-)$ & - & - & - & - & - & + & - & - & - & ND & - & - & - \\
\hline$\beta$-Galactosidase & - & (v) & $\mathrm{v}$ & $(-)$ & - & - & - & - & - & + & + & - & w & $\mathrm{ND}$ & - & - & - \\
\hline$\alpha$-Glucosidase & - & $(+)$ & $\mathrm{v}$ & $(+)$ & + & + & + & + & + & + & + & + & + & $\mathrm{ND}$ & + & - & + \\
\hline$\beta$-Glucosidase & - & (w) & - & (w) & + & - & w & - & w & - & - & - & - & $\mathrm{ND}$ & - & + & - \\
\hline Leucine arylamidase & - & $(+)$ & + & $(+)$ & + & + & + & + & + & + & + & - & + & $\mathrm{ND}$ & + & + & + \\
\hline
\end{tabular}




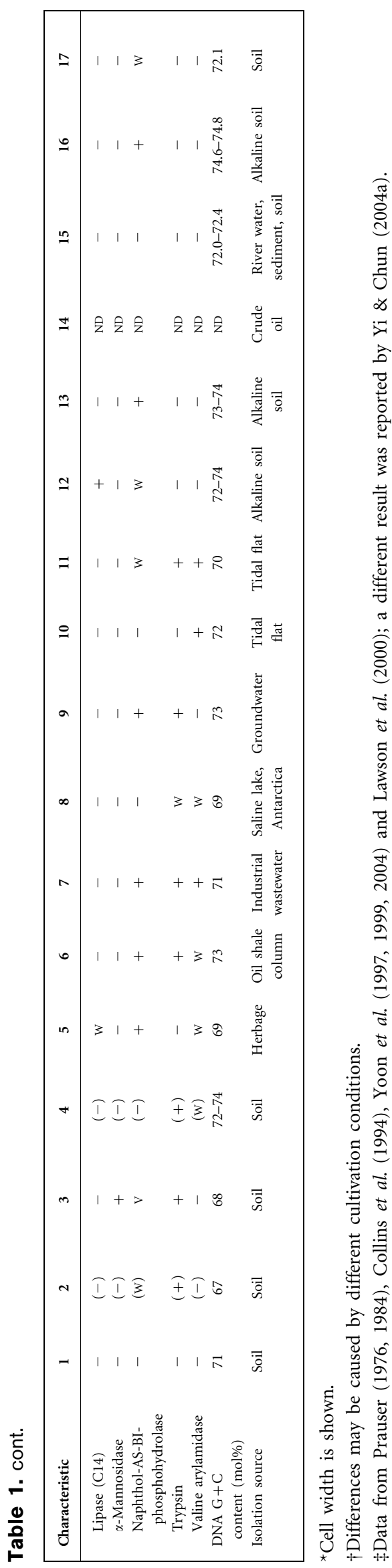

milarity values of $92.5-95.1 \%$ with respect to the type strains of other Nocardioides species and showed values of less than $92.1 \%$ sequence similarity with other species used in the phylogenetic analysis.

The chemotaxonomic properties supported the result of the monothetic phylogenetic classification suggesting that strain DS- $51^{\mathrm{T}}$ could be a member of the genus Nocardioides (Tamura \& Yokota, 1994; Yoon et al., 1997; Lawson et al., 2000; Urzì et al., 2000; Wang et al., 2001). The diagnostic diamino acid present in strain DS- $51^{\mathrm{T}}$ was LL-2,6diaminopimelic acid, which is characteristic of wall chemotype I sensu Lechevalier \& Lechevalier (1970). The predominant isoprenoid quinone detected in strain DS-51 ${ }^{\mathrm{T}}$ was a tetrahydrogenated menaquinone with eight isoprene units $\left[\mathrm{MK}-8\left(\mathrm{H}_{4}\right)\right]$. The cellular fatty acid profile (i.e. fatty acids representing $>1 \%$ of the total) comprised the following: branched fatty acids iso- $\mathrm{C}_{16: 0}(49.7 \%)$, anteiso- $\mathrm{C}_{17: 0}(4.9 \%)$, iso- $\mathrm{C}_{17: 0}(2.6 \%)$, iso- $\mathrm{C}_{18: 0}(2.3 \%)$, iso$\mathrm{C}_{15: 0}(1.6 \%)$ and iso- $\mathrm{C}_{16: 1}(2.3 \%)$; unsaturated fatty acids $\mathrm{C}_{18: 1} \omega 9 c(7.4 \%), \mathrm{C}_{17: 1} \omega 6 c(5.4 \%)$ and $\mathrm{C}_{17: 1} \omega 8 c(1.1 \%)$; straight-chain fatty acids $\mathrm{C}_{18: 0}(2.3 \%), \mathrm{C}_{17: 0}(1.9 \%)$ and $\mathrm{C}_{16: 0}(1.7 \%)$; 10 -methyl fatty acids $\mathrm{C}_{18: 0}(8.3 \%), \mathrm{C}_{17: 0}$ $(2.9 \%)$ and $\mathrm{C}_{16: 0}(1.8 \%)$, and summed feature $3\left(\mathrm{C}_{16: 1} \omega 7 c\right.$ and/or iso- $\left.\mathrm{C}_{15: 0} 2-\mathrm{OH}\right)(1.6 \%)$. This fatty acid profile is similar to those of Nocardioides species, although there are differences in the proportions of some fatty acids, perhaps because of differences in the extraction and cultivation conditions (Yoon et al., 1997, 1999, 2004, 2005a, b; Lawson et al., 2000; Yi \& Chun, 2004a, b; Kubota et al., 2005). The DNA G $+C$ content of strain DS- $51^{\mathrm{T}}$ was $71.1 \mathrm{~mol} \%$. The differential phenotypic properties of DS- $51^{\mathrm{T}}$, together with its phylogenetic distinctiveness, are sufficient to categorize the strain as a novel species of the genus Nocardioides (Stackebrandt \& Goebel, 1994; Table 1), for which the name Nocardioides insulae sp. nov. is proposed.

\section{Description of Nocardioides insulae sp. nov.}

Nocardioides insulae (in.su'lae. L. fem. gen. n. insulae of an island, referring to the source of isolation of the type strain).

Cells are aerobic, non-spore-forming, Gram-positive rods or cocci $(0.6-1.0 \times 1.0-6.0 \mu \mathrm{m})$ in the exponential phase of growth. Cells show rod-to-coccus morphogenesis from the early exponential phase to the stationary phase. Oxidasepositive. Colonies are circular, smooth, glistening, slightly convex, ivory in colour and $1.0-1.5 \mathrm{~mm}$ in diameter after 7 days incubation on NA at $30^{\circ} \mathrm{C}$. Neither substrate nor aerial mycelium is formed. Growth occurs at 10 and $34^{\circ} \mathrm{C}$, but not at 4 or $35^{\circ} \mathrm{C}$. The optimal $\mathrm{pH}$ for growth is 8.0 ; growth occurs at $\mathrm{pH} 6.5$, but not at $\mathrm{pH}$ 6.0. Growth occurs in the presence of $0-3 \%(\mathrm{w} / \mathrm{v}) \mathrm{NaCl}$, but is optimum in the absence of $\mathrm{NaCl}$. Arginine dihydrolase, lysine decarboxylase, ornithine decarboxylase and tryptophan deaminase activities are absent. $\mathrm{H}_{2} \mathrm{~S}$ and indole are not produced. Tweens 20, 40 and 60 are hydrolysed. Acetate, L-malate, pyruvate and L-glutamate are utilized as sole carbon and energy sources and salicin is weakly utilized, but citrate, succinate, 


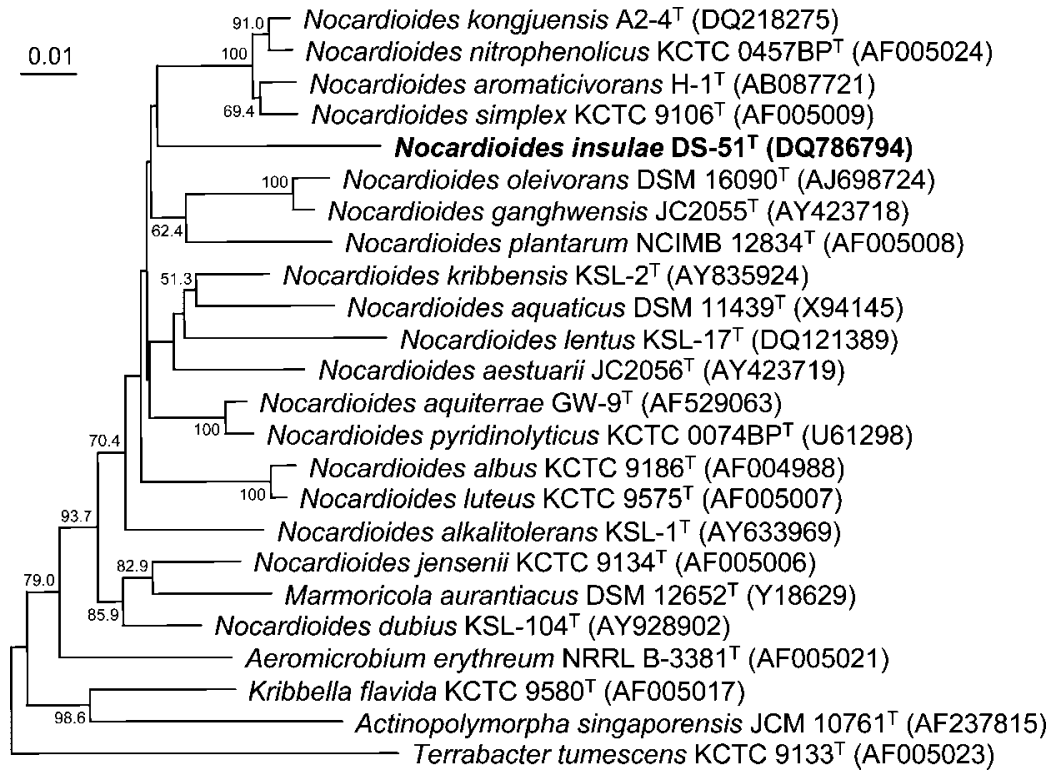

Fig. 1. Neighbour-joining phylogenetic tree, based on 16S rRNA gene sequences, showing the positions of strain $\mathrm{DS}-51^{\top}$ and some related taxa. Bootstrap values (expressed as percentages of 1000 replications) greater than $50 \%$ are shown at the branch points. Bar, 0.01 substitutions per nucleotide position.

benzoate and formate are not utilized. The cell-wall peptidoglycan contains LL-2,6-diaminopimelic acid as the diagnostic diamino acid. The predominant menaquinone is MK- $8\left(\mathrm{H}_{4}\right)$. The major fatty acid ( $>10 \%$ of total fatty acids) is iso- $\mathrm{C}_{16: 0}$. The DNA $\mathrm{G}+\mathrm{C}$ content is $71.1 \mathrm{~mol} \%$ (determined by HPLC). Other phenotypic characteristics are given in Table 1 .

The type strain, DS $-51^{\mathrm{T}}\left(=\mathrm{KCTC} 19180^{\mathrm{T}}=\mathrm{DSM} 17944^{\mathrm{T}}\right)$, was isolated from soil.

\section{Acknowledgements}

This work was supported by the 21C Frontier Program of Microbial Genomics and Applications (grant MG05-0401-2-0) from the Ministry of Science and Technology (MOST) of the Republic of Korea.

\section{References}

Collins, M. D., Dorsch, M. \& Stackebrandt, E. (1989). Transfer of Pimelobacter tumescens to Terrabacter gen. nov. as Terrabacter tumescens comb. nov. and of Pimelobacter jensenii to Nocardioides as Nocardioides jensenii comb. nov. Int J Syst Bacteriol 39, 1-6.

Collins, M. D., Cockcroft, S. \& Wallbanks, S. (1994). Phylogenetic analysis of a new LL-diaminopimelic acid-containing coryneform bacterium from herbage, Nocardioides plantarum sp. nov. Int J Syst Bacteriol 44, 523-526.

Komagata, K. \& Suzuki, K. (1987). Lipids and cell-wall analysis in bacterial systematics. Methods Microbiol 19, 161-203.

Kubota, M., Kawahara, K., Sekiya, K., Uchida, T., Hattori, Y., Futamata, H. \& Hiraishi, A. (2005). Nocardioides aromaticivorans sp. nov., a dibenzofuran-degrading bacterium isolated from dioxinpolluted environments. Syst Appl Microbiol 28, 165-174.

Lawson, P. A., Collins, M. D., Schumann, P., Tindall, B. J., Hirsch, P. \& Labrenz, M. (2000). New LL-diaminopimelic acid-containing actinomycetes from hypersaline, heliothermal and meromictic Antarctic Ekho Lake: Nocardioides aquaticus sp. nov. and Friedmanniella lacustris sp. nov. Syst Appl Microbiol 23, 219-229.
Lechevalier, M. P. \& Lechevalier, H. A. (1970). A critical evaluation of the genera of aerobic actinomycetes. In The Actinomycetales, pp. 393-405. Edited by H. Prauser. Jena: Gustav Fischer.

Prauser, H. (1976). Nocardioides, a new genus of the order Actinomycetales. Int J Syst Bacteriol 26, 58-65.

Prauser, H. (1984). Nocardioides luteus spec. nov. Z Allg Microbiol 24, 647-648.

Prauser, H. (1989). Genus Nocardioides Prauser 1976. In Bergey's Manual of Systematic Bacteriology, vol. 4, pp. 2371-2375. Edited by S. T. Williams, M. E. Sharpe \& J. G. Holt. Baltimore: Williams \& Wilkins.

Schippers, A., Schumann, P. \& Spröer, C. (2005). Nocardioides oleivorans sp. nov., a novel crude oil-degrading bacterium. Int J Syst Evol Microbiol 55, 1501-1504.

Stackebrandt, E. \& Goebel, B. M. (1994). Taxonomic note: a place for DNA-DNA reassociation and 16S rRNA sequence analysis in the present species definition in bacteriology. Int J Syst Bacteriol 44, 846-849.

Suzuki, K. \& Komagata, K. (1983). Pimelobacter gen. nov., a new genus of coryneform bacteria with LL-diaminopimelic acid in the cell wall. J Gen Appl Microbiol 29, 59-71.

Tamura, T. \& Yokota, A. (1994). Transfer of Nocardioides fastidiosa Collins and Stackebrandt 1989 to the genus Aeromicrobium as Aeromicrobium fastidiosum comb. nov. Int J Syst Bacteriol 44, 608-611.

Urzì, C., Salamone, P., Schumann, P. \& Stackebrandt, E. (2000). Marmoricola aurantiacus gen. nov., sp. nov., a coccoid member of the family Nocardioidaceae isolated from a marble statue. Int J Syst Evol Microbiol 50, 529-536.

Wang, Y. M., Zhang, Z. S., Xu, X. L., Ruan, J. S. \& Wang, Y. (2001). Actinopolymorpha singaporensis gen. nov., sp. nov., a novel actinomycete from the tropical rainforest of Singapore. Int $J$ Syst Evol Microbiol 51, 467-473.

Yi, H. \& Chun, J. (2004a). Nocardioides ganghwensis sp. nov., isolated from tidal flat sediment. Int J Syst Evol Microbiol 54, 1295-1299.

Yi, H. \& Chun, J. (2004b). Nocardioides aestuarii sp. nov., isolated from tidal flat sediment. Int J Syst Evol Microbiol 54, 2151-2154.

Yoon, J.-H., Rhee, S.-K., Lee, J.-S., Park, Y.-H. \& Lee, S. T. (1997). Nocardioides pyridinolyticus sp. nov., a pyridine-degrading bacterium isolated from the oxic zone of an oil shale column. Int J Syst Bacteriol 47, 933-938. 
Yoon, J.-H., Cho, Y.-G., Lee, S. T., Suzuki, K.-I., Nakase, T. \& Park, Y.-H. (1999). Nocardioides nitrophenolicus sp. nov., a p-nitrophenoldegrading bacterium. Int J Syst Bacteriol 49, 675-680.

Yoon, J.-H., Kim, I.-G., Kang, K. H., Oh, T.-K. \& Park, Y.-H. (2004). Nocardioides aquiterrae sp. nov., isolated from groundwater in Korea. Int J Syst Evol Microbiol 54, 71-75.

Yoon, J.-H., Kim, I.-G., Lee, M.-H., Lee, C.-H. \& Oh, T.-K. (2005a). Nocardioides alkalitolerans sp. nov., isolated from an alkaline serpentinite soil in Korea. Int J Syst Evol Microbiol 55, 809-814.
Yoon, J.-H., Kim, I.-G., Lee, M.-H. \& Oh, T.-K. (2005b). Nocardioides kribbensis sp. nov., isolated from an alkaline soil. Int J Syst Evol Microbiol 55, 1611-1614.

Yoon, J.-H., Lee, C.-H. \& Oh, T.-K. (2006a). Nocardioides lentus sp. nov., isolated from an alkaline soil. Int J Syst Evol Microbiol 56, 271-275.

Yoon, J.-H., Lee, J.-K., Jung, S.-Y., Kim, J.-A., Kim, H.-K. \& Oh, T.-K. (2006b). Nocardioides kongjuensis sp. nov., an $N$-acylhomoserine lactone-degrading bacterium. Int J Syst Evol Microbiol 56, 1783-1787. 